\title{
Comparison of divesified angles for evaluation of sagittal skeletal discrepancy in Bihar population: A cephalometric study
}

\author{
Amit Kumar ${ }^{1}$, Neal Bharat Kedia ${ }^{2}$, Amesh Golwara ${ }^{3}$, Monica Ritu Kedia ${ }^{4}$, Shikha Shalini ${ }^{5}$, Garima Verma ${ }^{6}$ \\ ${ }^{1,5,6}$ Consultant Orthodontist, ${ }^{2}$ Professor, ${ }^{3}$ Professor and Head, ${ }^{4}$ Reader, ${ }^{1,5,6}$ Consultant Orthodontist, Patna, Bihar, ${ }^{2-4}$ Dept. of Orthodontics, \\ ${ }^{2-4}$ Buddha Institute of Dental Sciences \& Hospital, Patna, Bihar, India
}

*Corresponding Author: Neal Bharat Kedia

Email: sky20083@ gmail.com

\begin{abstract}
Background: Varying number of lateral cephalometric analyses has emerged to analyse the skeletal relationships of jaw in the sagittal plane which is the most necessitate process in any kind of orthodontic diagnosis.

Aim: The present study was aimed to calculate the values of different angles (ANB angle, Wits appraisal, BETA angle, YEN angle, W angle and ASB angle) which further aid to assess the anterior-posterior jaw relationship on selected subjects of the local population of Bihar region.

Materials and Methods: The research sample comprised of lateral cephalograms of 90 subjects aged between 18-25 years with equal gender distribution from the Department of Orthodontics, Buddha Institute of Dental Science \& Hospital, Patna which were grouping further as Class I, II and III skeletal pattern (each consisted of 30 subjects).

Results: Mean values for each angle was derived for Bihar population of all three classes and different angles were compared with other previous studies.

Conclusion: Apart from certain regular parameters which showed statistically significant differences between subjects of the Bihar population, a new angle i.e. ASB is also taken into consideration which certainly can help in orthodontic diagnosis and treatment planning. Further studies are needed on large scale to establish data for the particular population.
\end{abstract}

Keywords: ANB angle, ASB angle, Beta angle, Wangle, Wits appraisal, Yen angle.

\section{Introduction}

In diagnosis and treatment planning of any orthodontic patient the cephalometric analysis is an indispensable part which is based on varied number of angular and linear measurements. Wylie in 1947 recommended jaws inspection in the sagittal plane as a principal step. ${ }^{1}$ Research has been continued, and in 2004 Baik and Ververidou proposed some facts about orthodontic diagnosis and treatment planning which further guide the sagittal apical base relationship. Any kind of cephalometric analysis basically comprised of both angular and linear measurements which assist the clinicians in interpretation of antero- posterior discrepancies and authorize the most appropriate treatment plan. ${ }^{2}$ Whichever is the cephalometric analysis they have shortcomings and limitations, discussed in detail by Moyers et al. ${ }^{3}$

Most advisable auxiliary measurement to define the apical base relationship would be independent of cranial reference planes or dental occlusion. Although, the ANB angle is most acclaimed and conducive, however point $\mathrm{A}$ and $\mathrm{B}$ can be altered by growth and dentoalveolar remodeling during the commencement of orthodontic treatment as also reported by many research papers. Nonetheless, growth also affects the location of nasion point (N) and any transference of nasion will directly affect the ANB angle. ${ }^{4}$ Jacobson proposed the use of Wits appraisal as an alternative, reason being that the rotational transition of the jaws and position of the nasion point has a direct impact on the value of ANB angle. ${ }^{5}$ Wits appraisal is defined as the distance between the lines drawn from the $\mathrm{A}$ and $\mathrm{B}$ points coming perpendicular to occlusal plane. Contrary to other parameters, Wits appraisal is not related to the skull base and nasion point. Though, the occlusal plane which defines its value can easily be altered by tooth eruption and dental development. ${ }^{6-8}$

Beta angle has been identified as a new perspective for assessing sagittal discrepancies which neither depends on any cranial landmarks nor on occlusion plane and can be productively used whenever previously established measurements, such as the ANB angle or the Wits appraisal, cannot be calculated precisely. Beta angle employ three skeletal landmarks - point $\mathrm{A}$, point $\mathrm{B}$, and point $\mathrm{C}$ (the apparent axis of the condyle). It is formed between A-B line and point A perpendicular to C-B line. ${ }^{9}$ Although, it assesses sagittal discrepancies precisely, but at times point A and B are difficult to locate and in some situations, either the condyle is not clearly visible or the actual site can be affected by growth or treatment (Holdway, 1970). ${ }^{10}$

Further after continuation of valuable researches, Yen angle ${ }^{11}$ and $\mathrm{W}$ angle ${ }^{12}$ are introduced as new analytical method, which utilize stable landmarks like Sella, M point and $G$ points. The Yen (2009) angle uses three reference points: S, midpoint of the sella-turcica; $\mathrm{M}$, midpoint of the premaxilla; and $\mathrm{G}$, center of the largest circle that is tangent to the internal inferior, anterior, and posterior surfaces of the mandibular symphysis. However, morphological landmarks appear to be more reliable, constructed points may in some instances epitomize in a superior way to reveal the accurate traits of the underlying skeletal pattern. When S, M, and G are connected, they form the Yen angle, which is measured at $\mathrm{M}$. This angle is unaltered by any growth transitional 
phases and can be straightforwardly used in the mixed dentition. ${ }^{11}$

Further Bhad et al raised a point that since it measures an angle between line SM and MG, and rotation of jaw can occur either due to normal development or as the result of orthodontic treatment which can mask true basal dysplasia. ${ }^{12}$ To conquer these issues, a new angle named as W angle was introduced by Bhad et al (2011) for assessing the skeletal discrepancy between maxilla and mandible in the sagittal plane. It employ three skeletal landmarks- point $S$, point $M$, and point $G$ which measuring the $W$ angle between the perpendicular line from point $\mathrm{M}$ to $\mathrm{S}-\mathrm{G}$ line and the $\mathrm{M}-\mathrm{G}$ line. The reference of $\mathrm{W}$ angle provides the advantage to remain relatively stable even when the jaws are rotated or growing vertically. It is a consequence of modification of S-G line along with jaw rotation. ${ }^{12}$

Silverman (1957) suggested ASB as an advanced variable in defining and formulating the norms for skeletal sagittal discrepancy. It is defined as the angle between A-S line and the S-B line where sella is taken as a reference point because it is a fixed cranial landmark in skull and which can be conveniently traceable on the lateral cephalogram. ${ }^{13}$

The purpose of present research is to assess and constitute the norms for varied cephalometric angles i.e. ANB angle, Wits appraisal, Beta angle, $\mathrm{W}$ angle, Yen angle and ASB angle in defining antero-posterior apical base discrepancy for local population of Bihar.

\section{Materials and Methods}

90 subjects (with equal gender distribution) in age range of 18-25 years were included in the present study group who reported at the Department of Orthodontics and Dentofacial Orthopedics, Buddha Institute of Dental Science \& Hospital, Patna as well as students studying in mentioned college were taken to maintain uniformity of the sample. All the members were belonging to Bihar in origin. Thorough medical history was taken regarding any previous major illness in childhood that might have affected the growth. The patients having such history were excluded from the study. Sample was grouped into Class I, Class II and Class III based on the clinical profile and dental occlusion pattern. Class I skeletal pattern confirmation by ANB angle which is 1-3 and Wits appraisal $1 \mathrm{~mm}$ (male), $0 \mathrm{~mm}$ (female). All the study participants were elucidated about the need if undergoing through clinical and radiographic investigation and consent form were taken.

Lateral cephalograms were taken for each subject on the Vatech PaX- 400 cephalometric machine in a natural head position, with the teeth in maximum intercuspation and lip relaxed. The film was exposed while operating the cephalostat at a constant of $74 \mathrm{KVP}, 12 \mathrm{Ma}$ and $0.8 \mathrm{sec}$ film exposure time. All the exposed film were developed and fixed manually by a single technician using standard procedure. All lateral radiographs were traced with the help of X-ray viewer on a transparent cellulose acetate sheet of 0.003 " thickness with sharp $3 \mathrm{H}$ pencil and landmarks were identified.

\section{Results}

The sample selected was further scrutinized by the 3 independent observers and 90 subjects were selected between the age group of 18 to 25 years with equal gender distribution for the cephalometric database of the present research. All the subjects were selected with equal distribution among Class I, Class II and Class III skeletal pattern and within the inclusion criteria discussed earlier. The results were obtained and statistically analyzed to assess the values (ANB angle, Wits apprasial, Beta angle, Yen angle, W angle and ASB angle) of selected population. Our results were also compared with other similar study groups to further evaluate the acceptance of these.

Table 1 shows the descriptive statistics of ANB angle, Wits appraisal, Beta angle, Yen angle, W angle and ASB angle for sample of 90 subjects among three classes of 30 each as Class I, Class II, Class III. The mean values along with standard deviation for ANB angle, Wits appraisal, Beta angle, Yen angle, $\mathrm{W}$ angle and ASB angle in different classes are also summarized in Fig. 1A, 1B, 1C, 1D, 1E and $1 \mathrm{~F}$ respectively.

Table 2 summarizes the coefficient of variation of the selected parameters in different study groups. Results depict that ANB angle and Wits appraisal shows high degree of variation as compared to other parameters which further confined the result and suggest that the values of ANB angle, Wits, Beta angle, Yen angle, W angle and ASB angle are more consistent and reliable as compared to ANB angle and Wits appraisal.

Table 3 exemplifies the correlation coefficient among all the sagittal jaw markers in different classes. Results depict significant correlation of ANB angle with Beta angle and Yen angle in Class I group $(\mathrm{p} \leq 0.005)$. Surprisingly, in Class II group ANB angle has non significant relation with all other parameters $(p \geq 0.005)$. Whereas in Class III group, ANB angle has the significant relation with all other parameters $(\mathrm{p} \leq 0.005)$.

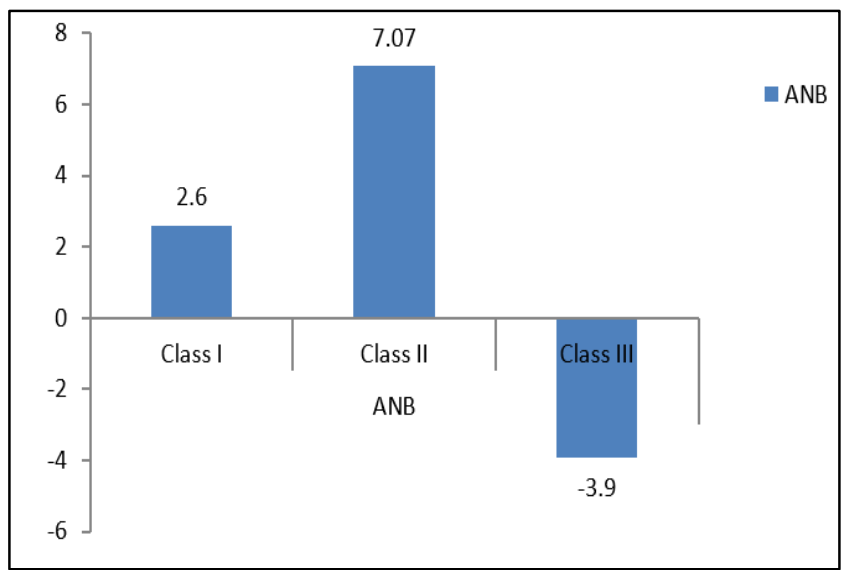

Fig. 1A: ANB 

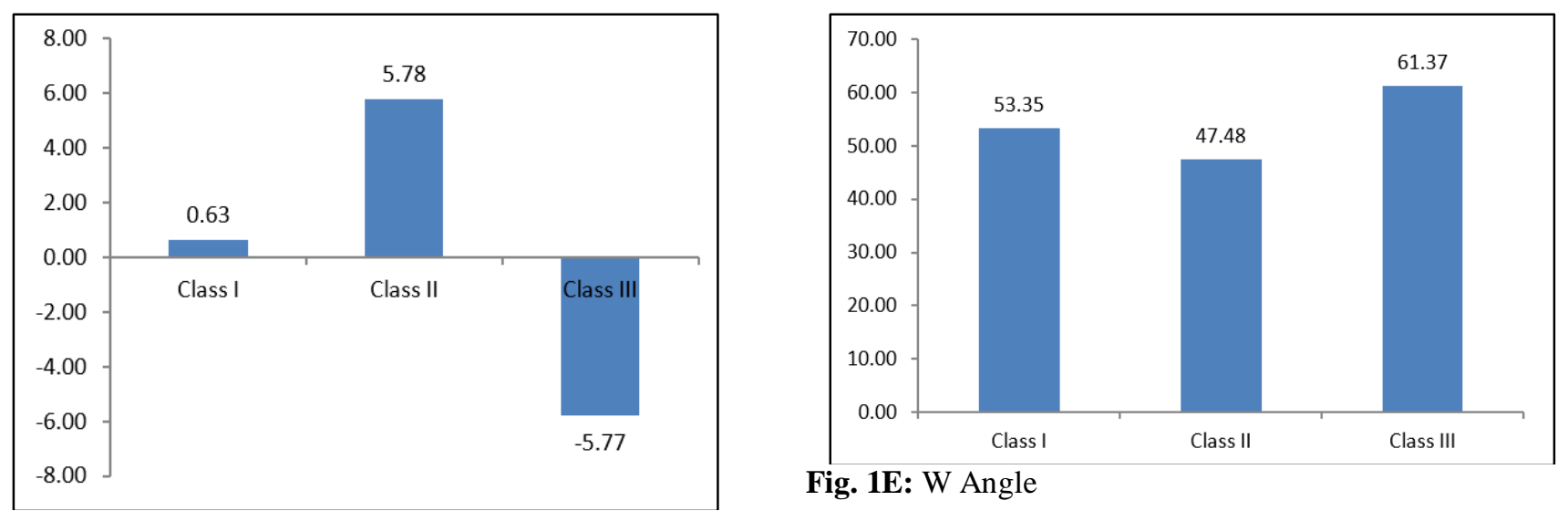

Fig. 1B: WITS

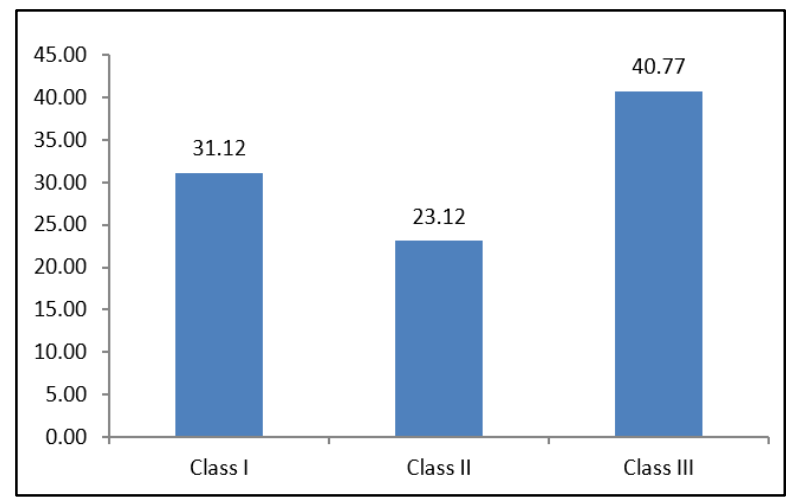

Fig. 1C: BETA

Fig. 1E: W Angle

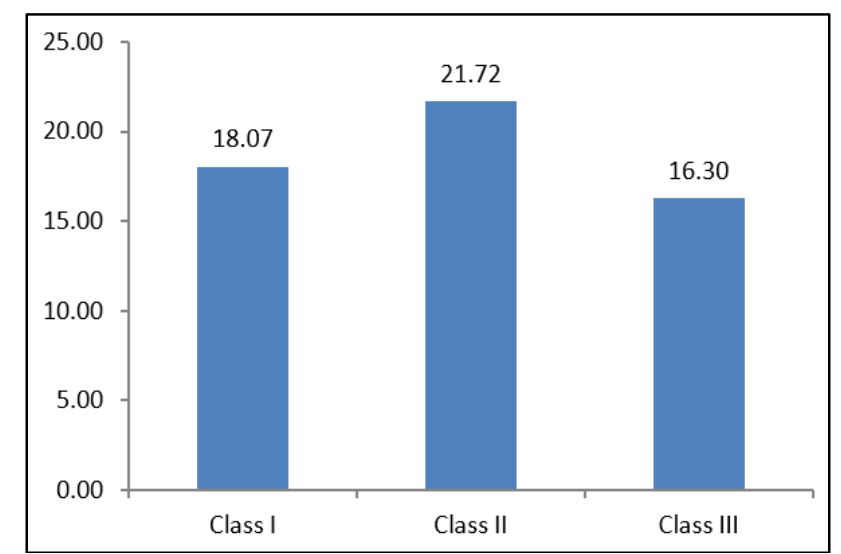

Fig 1F: ASB

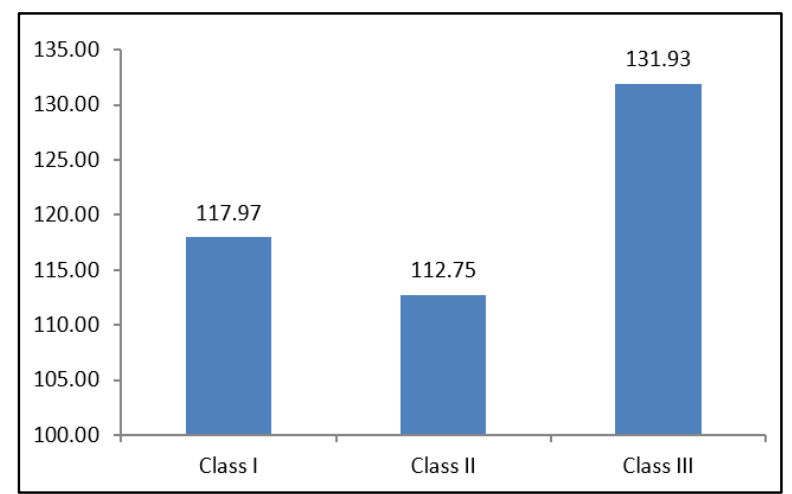

Fig. 1D: YEN

Table 1: Comparison of Range, Mean, Standard deviation and Coefficient of Variation\%

\begin{tabular}{|l|l|c|c|c|c|}
\hline \multirow{4}{*}{ ANB } & Classification & Range & Mean & $\mathbf{\pm S D}$ & CV\% \\
\cline { 2 - 6 } & Class I & $0-3$ & 2.6 & 0.90 & 34.54 \\
\cline { 2 - 6 } & Class II & $>3$ & 7.07 & 2.17 & 30.77 \\
\cline { 2 - 6 } & Class III & $<1$ & -3.9 & 2.67 & -68.51 \\
\hline \multirow{4}{*}{ WITS } & Class I & $0-1$ & 0.63 & 0.74 & 116.98 \\
\cline { 2 - 6 } & Class II & $>1$ & 5.78 & 2.16 & 37.35 \\
\cline { 2 - 6 } & Class III & $<0$ & -5.77 & 2.88 & -50.00 \\
\hline \multirow{3}{*}{ YEN } & Class I & $<27$ & 31.12 & 2.79 & 8.97 \\
\cline { 2 - 6 } & Class II & $>35$ & 23.12 & 3.81 & 16.49 \\
\cline { 2 - 6 } & Class III & $36-45$ & 40.77 & 8.03 & 19.70 \\
\cline { 2 - 6 } & Class I & $117-123$ & 117.97 & 19.30 & 16.36 \\
\cline { 2 - 6 } & Class II & $<117$ & 112.75 & 3.29 & 2.92 \\
\hline
\end{tabular}




\begin{tabular}{|l|l|c|c|c|c|}
\hline & Class III & $>123$ & 131.93 & 4.97 & 3.76 \\
\hline \multirow{5}{*}{ W ANGLE } & Class I & $51-55$ & 53.35 & 1.75 & 3.27 \\
\cline { 2 - 6 } & Class II & $<51$ & 47.48 & 4.69 & 9.87 \\
\cline { 2 - 6 } & Class III & $>55$ & 61.37 & 3.07 & 5.00 \\
\hline \multirow{3}{*}{ CSB } & Class I & -- & 18.07 & 3.47 & 19.20 \\
\cline { 2 - 6 } & Class II & -- & 21.72 & 2.28 & 10.50 \\
\cline { 2 - 6 } & Class III & -- & 16.30 & 2.37 & 14.5 \\
\hline
\end{tabular}

Table 2: Coefficient of Variation\%

\begin{tabular}{|l|c|c|c|c|c|c|}
\hline & ANB & WITS & BETA & YAN & W ANGLE & ASB \\
\hline Class I & 34.54 & 116.98 & 8.97 & 16.36 & 3.27 & 19.20 \\
\hline Class II & 30.7 & 37.35 & 16.49 & 2.92 & 9.87 & 10.50 \\
\hline Class III & -68.51 & -50.00 & 19.70 & 3.76 & 5.00 & 14.55 \\
\hline
\end{tabular}

Table 3: Class wise correlation coefficient among all the sagittal jaw markers

\begin{tabular}{|l|c|c|c|c|}
\hline Parameters & Classification & Correlation cofficient & $\mathbf{r}^{\mathbf{2}}$ & P Value \\
\hline Class I & ANB vs WITS & 0.7685 & 0.6127 & 0.0261 \\
\cline { 2 - 5 } & ANB vs BETA & -0.6182 & 0.8524 & 0.0067 \\
\cline { 2 - 5 } & ANB vs YEN & -0.9124 & 0.8962 & 0.0021 \\
\cline { 2 - 5 } & ANB vs W angle & 0.7352 & 0.8967 & 0.0043 \\
\cline { 2 - 5 } & ANB vs ASB & 0.4125 & 0.8926 & 0.0584 \\
\hline Class II & ANB vs WITS & 0.6128 & 0.4152 & 0.0081 \\
\cline { 2 - 5 } & ANB vs BETA & -0.3985 & 0.7825 & 0.0638 \\
\cline { 2 - 5 } & ANB vs YEN & -0.3569 & 0.9812 & 0.5129 \\
\cline { 2 - 5 } & ANB vs W angle & -0.0425 & 0.8641 & 0.3125 \\
\cline { 2 - 5 } & ANB vs ASB & 0.6325 & 0.8912 & 0.0113 \\
\hline Class III & ANB vs WITS & 0.7814 & 0.2151 & 0.0019 \\
\cline { 2 - 5 } & ANB vs BETA & -0.7512 & 0.6954 & 0.0039 \\
\cline { 2 - 5 } & ANB vs YEN & -0.8625 & 0.9365 & 0.0041 \\
\cline { 2 - 5 } & ANB vs W angle & -0.8623 & 0.9362 & 0.0032 \\
\cline { 2 - 5 } & ANB vs ASB & 0.8629 & 0.8659 & 0.0049 \\
\hline
\end{tabular}

\section{Discussion}

Lateral cephalogram is the key tool for the assessment of antero- posterior jaw relationship which further guides the diagnosis and treatment planning of any orthodontic case. Both angular and linear measurements have been incorporated into cephalometric analysis to evaluate sagittal jaw relationship. Various studies have been conducted to assess the reliability of these parameters which can be used uniformly with accurate and reproducible results. ${ }^{14}$

Formerly, ANB angle was widely used parameter but due to certain drawbacks like during growth the position of nasion is not fixed and any displacement of nasion will directly affect the ANB angle, furthermore, it is not a favourable one now a days. Rotation of jaw either by normal growth pattern or orthodontic treatment can directly alter the value of ANB angle. , $^{5,10,14}$

Wits appraisal came into existence as a substitute for ANB angle which is independent of cranial landmarks and even does not get affected by rotation of the jaws but this one also has a drawback of difficulty encounter in identifying the functional occlusal plane, which can be unfeasible at times. ${ }^{15}$

Further, to overcome these demerits, a new Beta angle was introduced. It makes use of 3 points located on the jaws- point A, point $\mathrm{B}$, and the apparent axis of the condyle (point C), so apparently any alteration in this angle reflect only when there are changes occur within the jaws. Contrary to ANB angle, the configuration of Beta angle gives it the advantage to remain relatively stable even when the jaws are rotated. For the reason being, clinicians might hesitate to use Beta angle is because tracing the condyle and locating its center accurately is a tedious process. ${ }^{14}$

After so many shortfalls of previous parameters, W angle and Yen angle were introduced which do not employ $\mathrm{A}$ and $\mathrm{B}$ points as skeletal landmarks, which further affected by local remodelling due to orthodontic treatment. ${ }^{11,12}$

In the present study we traced the lateral cephalogram of 90 individuals of different class groups. An attempt was made to inspect and propose the standardizing values of ANB angle, Wits appraisal, Beta angle, Yen angle, W angle and a new angle, ASB angle among local Bihar population. The mean values of Wits appraisal, ANB angle, Beta angle, Yen angle, $\mathrm{W}$ angle and ASB angle in each class differ significantly from each other.

The mean value for ANB angle in Bihar population in Class 1 group is $2.6^{0}$ which is very approximate to ANB mean value of Reidal ${ }^{16} 3.4^{0}$ and Jarvinen ${ }^{17} 2.9^{0}$. Contrary to this, Walker and Kowalski ${ }^{18}$ found mean ANB value of $4.5^{0}$ which is comparatively higher. In the present research, the mean value for ANB angle in Class II and Class III are 
$7.06 \pm 2.1$ and $-3.9 \pm 2.6$ respectively. Our results hypothesize the fact that the ANB angle values are not significant in all three classes. This is in concordance with other studies conducted by Brown (1981), ${ }^{6}$ Chang $(1987)^{8}$ and Jacobson (1975), ${ }^{5}$ who claimed that any change in the SN plane would affect the ANB angle. This is also promoted by Rotberg et al (1980) who stated that nasion usually moves in anterior and slightly superior direction because of the growth increments on the cranial base plane passing through sella and nasion. Therefore, during usage of ANB angle, aspects should be taken into consideration are patient's age, growth rotation of the jaws, vertical growth, and the length of the anterior cranial base (AP position of $\mathrm{N}$ ), which further makes the interpretation of this angle copious. ${ }^{5}$

The mean value for Wits appraisal in Bihar population in Class 1 is 0.63 , which is much closed to the Wits value measure by Jarvinen ${ }^{17}-0.6 \pm 2.9$ and Jacobson ${ }^{5} 1.07 \pm 1.77$. The mean value for Wits appraisal in Class II and Class III are $5.78 \pm 2.1$ and $-5.77 \pm 2.8$ respectively. Similar to ANB angle, Wits value is also not satisfactorily significant among three groups. This is in concordance with Moore et al $(1989)^{20}$ and Ishikawa et al $(2000)^{21}$ who describe the fact that although Wits appraisal is not affected by landmarks or jaw rotations but it is difficult to identify the functional occlusal plane, which can be impossible at times, especially in mixed dentition.. Any alteration in angulation of occlusal plane extremely influences the positions of A, B and thereby Wits appraisal reading.

The mean value for Beta angle in Bihar population in Class I is $31.12^{0}$ and is proximate to Baik and Ververidou ${ }^{2}$ mean Beta values of $31.1 \pm 2.0$. The mean value for Beta angle in Class II and Class III are $23.12 \pm 3.8$ and $40.77 \pm 8.0$ respectively. Beta angle mean values were statistically significant among Class I and Class III groups in the present research work. This is similar to Biak and Ververidou ${ }^{2}$ where they presented the fact that Beta angle does not depend on cranial landmarks or the functional occlusal plane and remain relatively stable even when the jaws are rotated. Another advantage of Beta angle is that it can be used in consecutive comparisons throughout orthodontic treatment because it reflects accurate changes of the sagittal relationship of the jaws, which might be due to growth or orthodontic or orthognathic intervention. But there is still a point of worry that tracing the condyle and locating its center is difficult. ${ }^{11}$

The mean value for Yen angle in Bihar population in Class $I$ is $117^{\circ}$ and is proximate to Yen angle values of Neela et al study ${ }^{11} 120.5^{0} \pm 2.9^{0}$. The mean value for Yen angle in Class II and Class III are 112 \pm 3.2 and $131 \pm 4.9$ respectively. Present research showed that Yen angle mean values were statistically significant in Class II and Class III among three groups. This is similar to study done by Neela et al $(2009)^{11}$ establishing the fact that Yen angle depend on stable points like S-midpoint of sella turcica, M- midpoint of pre-maxilla and G- center of largest circle that is tangent to the internal inferior, anterior, and posterior surfaces of the mandibular symphysis and hence relatively uninfluenced by growth phases and can easily be used in mixed dentition.
But since it measures an angle between line SM and MG, rotation of jaw either due to growth or orthodontic treatment can mask true basal dysplasia, similar to ANB angle.

The mean value for $\mathrm{W}$ angle in Bihar population in Class 1 is $53.3^{0}$ and is proximate to mean value of Bhad et al study $^{12} 52.2 \pm 2.3$. The mean value for $\mathrm{W}$ angle in Class 1 and Class III are $47.4 \pm 4.6$ and $61.3 \pm 3.0$ respectively. Surprisingly, the present study showed statistically significant $\mathrm{W}$ angle mean values among all three classes. Bhad et $\mathrm{al}^{12}$ found similar results and stated that $\mathrm{W}$ angle does not depend on unstable landmarks or the functional occlusal plane. The positional effect of $\mathrm{W}$ angle also has the advantage to remain relatively stable even when the jaws are rotated or growing vertically this is because of rotation of the S-G line along with jaw rotation, which carries the perpendicular from point $\mathrm{M}$ with it. Therefore, measurement of $\mathrm{W}$ angle is precise sagittal parameter in skeletal patterns with clockwise or counterclockwise rotation of the jaws as well as during transitional period when vertical facial growth is taking place.

The mean value for ASB angle in Bihar population in different classes are $18.07 \pm 3.4,21.72 \pm 2.2$ and $16.3 \pm 2.3$ for Class I, Class II, and Class III respectively.

With the enormous documentation stated in the present research about the fact that points A and B are affected by remodeling changes during growth and are influenced by orthodontic treatment, ${ }^{22,23}$ there is a heightened need of an hour for a technique or measurement that will abolish or reduce to minimum the inaccuracies in the presently used parameters. Common difficulties encountered in defining the location of point A and point B could directly affect the Wits appraisal, ANB angle and Beta angle calculation methods. ${ }^{24}$ As hard tissue land marks are used for determining YEN angle and $\mathrm{W}$ angle (Sella, constructed Points $M, G$ ) which further represent the exact nature of underlying skeletal pattern and therefore, less affected by remodeling changes during orthodontic treatment. ${ }^{11}$ So, these can be used substantially over other previous parameters. It is possible that YEN angle, $\mathrm{W}$ angle and ASB angle can be affected by jaw rotation as it depends on cranial landmark. This aspect is not studied in the present research and needs to be evaluated in further studied. Further studies are needed on large scale to establish data for the particular population. The present pilot study would plan the way for more intensive research in this direction.

\section{Conclusion}

Apart from certain regular parameters which showed statistically significant differences between subjects of the Bihar population, a new angle i.e. ASB is also taken into consideration which certainly can help in orthodontic diagnosis and treatment planning. Further studies are needed on large scale to establish data for the particular population.

\section{Source of Funding}

None. 


\section{Conflict of Interest}

None.

\section{References}

1. Wylie WL. The assessment of anteroposterior dysplasia. Angle Orthod. 1947;17(3):97-109.

2. Baik CY, Ververidou M. A new approach of assessing sagittal discrepencies: The Beta Angle. Am J Orthod Dentofacial Orthop. 2004;126(1):100-5.

3. Moyers RE, Bookstein FL, Guire KE. The concept of pattern in craniofacial growth. Am J Orthod. 1979;76(2):136-48.

4. Ferrazzini G. Critical evolution of the ANB angle. Am J Orthod. 1976;69(6):620-6.

5. Jacobson A. The 'Wits' appraisal of jaw disharmony. Am J Orthod. 1975;67:125-38.

6. Brown M. Eight methods of analysing a cephalogram to establish anteroposterior skeletal discrepancy. Br J Orthod. 1981;8:139-46.

7. Hussels W, Nanda RS. Analysis of factors affecting angle ANB. Am J Orthod. 1984;85:411-23.

8. Chang HP. Assessment of anteroposterior jaw relationship. Am J Orthod Dentofacial Orthop. 1987;92:117-22.

9. Singh G, Verma S, Singh DP, Yadav SK, Yadav AB Correlation of beta angle with antero-posterior dysplasia indicators and FMA: An institution based cephalometric study. J Clin Diagnostic Res. 2016;10(11):ZC75-ZC8.

10. Holdway RA. Changes in relationship of points A and B during orthodontic treatment. Am J Orthod Dentofac Orthop. 1956;42(3)176-93.

11. Neela PK, Mascarenhas R, Husain A. A New sagittal dysplasia indicator; The Yen angle. World J Orthod. 2009;10(2):147-51.

12. Bhad WA, Nayak S, Doshi UH. A new approach of assessing sagittal dysplasia- the W angle. Eur J Orthod. 2013;35(1):66-70

13. Kim YH, Vietas JJ. Anteroposterior dysplasia indicator, an adjunct to cephalometric different diagnosis. Am J Orthod. 1978;73(6):619-33
14. Potode NB, Bajaj TD, Verulkar AA, Wankhade SB, Lohakpure RA, Sangatani JK. Norms for anterior-posterior assessment of jaw relationship in Maharashtra population. Int $J$ Orthod Rehabil. 2018;9:141-4.

15. Haynes $\mathrm{S}$, Chau MN. The reproducibility and repeatability of the wits analysis. Am J Orthod Dentofacial Orthop. 1995;107:640-7.

16. Riedel RA. The relation of maxillary structures to cranium in malocclusion and in normal occlusion. Angle Orthod. 1952;22(3):142-5.

17. Jarvinen S. Relation of the SNA angle to the saddle angle. Am J Orthod. 1980;78(6):670-3.

18. Walker GF, Kowalski CJ. The Distribution of the ANB angle in "normal" individuals. Angle Orthod. 1971;41(4):332-5.

19. Rotberg S, Fried N, Kane J, Shapiro E. Predicting the Wits appraisal from the ANB angle. Am J Orthod. 1980;77(6)636-42.

20. Moore RN, DuBois LM, Boice PA, Igel KA. The accuracy of measuring condylion location. Am J Orthod Dentofacial Orthop. 1989;95(4):344-7.

21. Ishikawa H, Nakamura S, Iwasaki H, Kitazawa S. Seven parameters describing anteroposterior jaw relationships: Post pubertal prediction accuracy and inter changeability. Am J Orthod Dentofacial Orthop. 2000;117(6):714-20.

22. Beatty EJ. A modified technique for evaluating apical base relationships. Am J Orthod. 1975;68(3):303-15.

23. Bhatia SN, Akpabio TA. A correlation study of two method of assessing skeletal pattern. Br J Orthod. 1979;6(4)187-93.

24. Roth R. The Wits appraisal, its skeletal and dentoalveolar background. Eur J Orthod. 1982;4:21-8.

How to cite this article: Kumar A, Kedia NB, Golwara A, Kedia MR, Shalini S, Verma G. Comparison of divesified angles for evaluation of sagittal skeletal discrepancy in Bihar population: A cephalometric study. Int J Oral Health Dent 2020;6(1):12-7. 